\title{
Association between uric acid lowering and renal function progression: a longitudinal study
}

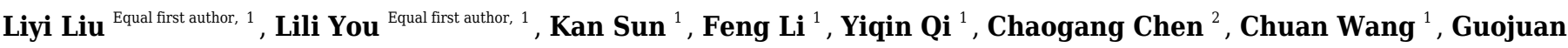 \\ Lao $^{1}$, Shengneng Xue ${ }^{1}$, Juying Tang ${ }^{1}$, Na Li ${ }^{1}$, Wanting Feng ${ }^{1}$, Chuan Yang ${ }^{1}$, Mingtong Xu ${ }^{1}, \operatorname{Yan~Li~}^{1}, \operatorname{Li~Yan}^{1}$, \\ Meng Ren ${ }^{\text {Corresp., } 1}$, Diaozhu Lin ${ }^{\text {Corresp. } 1}$ \\ 1 Department of Endocrinology, Sun Yat-Sen Memorial Hospital, Sun Yat-sen University, Guangzhou, China \\ 2 Department of Clinical Nutrition, Sun Yat-sen Memorial Hospital, Sun Yat-sen University, Guangzhou, China \\ Corresponding Authors: Meng Ren, Diaozhu Lin \\ Email address: renmeng80@139.com, lindzh6@mail.sysu.edu.cn
}

Background : This study aimed to explore the association between uric acid lowering and renal function.

Materials and Methods: We conducted a population-based cohort study with 1534 subjects for 4 years from 2012 to 2016. The population was divided into four groups according to the interquartile range of changes in serum uric acid with quartile 1 representing lower quarter. Renal function decline was defined as eGFR decreased more than $10 \%$ from baseline in 2016. Renal function improvement was defined as eGFR increased more than $10 \%$ from baseline in 2016. Cox regression analysis was used to calculate the hazard ratio (HR) and $95 \%$ confidence interval $(\mathrm{Cl})$.

Results: In the adjusted Cox regression models, compared to quartile 4, quartile 1 ( $\mathrm{HR}=0.64,95 \% \mathrm{Cl}$ : $0.49-0.85$ ), quartile $2(\mathrm{HR}=0.65,95 \% \mathrm{Cl}: 0.50-0.84)$ and quartile $3(\mathrm{HR}=0.75,95 \% \mathrm{Cl}: 0.58-0.96)$ have reduced risk of renal function decline. An increasing hazard ratio of renal function improvement was shown in quartile $1(\mathrm{HR}=2.27,95 \% \mathrm{Cl}: 1.45-3.57)$ and quartile $2(\mathrm{HR}=1.78,95 \% \mathrm{Cl}: 1.17-2.69)$ compared with quartile 4.

Conclusions: Uric acid lowering is associated with changes in renal function. The management of serum uric acid should receive attention in clinical practice and is supposed to be part of the treatment of chronic kidney disease. 
1 Association between uric acid lowering and renal

\section{2 function progression: a longitudinal study}

5 Liyi Liu ${ }^{1 *}$ Lili You ${ }^{1 *}$ Kan Sun ${ }^{1}$ Feng Li $^{1}$ YiQin Qi ${ }^{1}$ Chaogang Chen ${ }^{2}$ Chuan Wang ${ }^{1}$ Guojuan Lao ${ }^{1}$

6 Shengneng Xue ${ }^{1}$ Juying Tang ${ }^{1} \mathrm{Na} \mathrm{Li}^{1}$ Wanting Feng ${ }^{1}$ Chuan Yang ${ }^{1}$ Mingtong $\mathrm{Xu}^{1} \mathrm{Yan}^{1}{ }^{1}$

$7 \quad$ Li Yan ${ }^{1}$ Meng Ren ${ }^{1 \#}$ Diaozhu Lin ${ }^{1 \#}$

8

91 Department of Endocrinology, Sun Yat-sen Memorial Hospital, Sun Yat-sen University

10 Guangzhou, People’s Republic of China

$11^{2}$ Department of Clinical Nutrition, Sun Yat-sen Memorial Hospital, Sun Yat-sen University,

12 Guangzhou, People’s Republic of China

14 Corresponding Author:

15 Meng Ren ${ }^{1}$

16107 Yanjiang West Road, Guangzhou, People’s Republic of China

17 Email address: corresponding author renmeng80@139.com

18 Diaozhu Lin ${ }^{1}$

19107 Yanjiang West Road, Guangzhou, People’s Republic of China

20 Email address: lindzh6@mail.sysu.edu.cn 


\section{Abstract}

22 Background: This study aimed to explore the association between uric acid lowering and renal

23 function.

24 Materials and Methods: We conducted a population-based cohort study with 1534 subjects for

254 years from 2012 to 2016. The population was divided into four groups according to the

26 interquartile range of changes in serum uric acid with quartile 1 representing lower quarter. Renal

27 function decline was defined as eGFR decreased more than 10\% from baseline in 2016. Renal

28 function improvement was defined as eGFR increased more than 10\% from baseline in 2016.

29 Cox regression analysis was used to calculate the hazard ratio (HR) and 95\% confidence interval 30 (CI).

31 Results: In the adjusted Cox regression models, compared to quartile 4, quartile 1 (HR $=0.64$,

32 95\% CI: 0.49 - 0.85), quartile $2(\mathrm{HR}=0.65$, 95\% CI: $0.50-0.84)$ and quartile $3(\mathrm{HR}=0.75$, 95\%

33 CI: 0.58 - 0.96) have reduced risk of renal function decline. An increasing hazard ratio of renal

34 function improvement was shown in quartile $1(\mathrm{HR}=2.27,95 \% \mathrm{CI}: 1.45-3.57)$ and quartile 2

$35 \quad(\mathrm{HR}=1.78,95 \% \mathrm{CI}: 1.17-2.69)$ compared with quartile 4.

36 Conclusions: Uric acid lowering is associated with changes in renal function. The management

37 of serum uric acid should receive attention in clinical practice and is supposed to be part of the 38 treatment of chronic kidney disease.

\section{Introduction}

41 Hyperuricemia (HUA) is a disease with a high incidence of $13.3 \%$ in China in the years from

422000 to $2014^{[1]}$. Hyperuricemia is an independent risk factor for hypertension ${ }^{[2,3]}$, diabetes ${ }^{[4,5]}$,

43 cardiovascular disease ${ }^{[6-8]}$ and renal disease ${ }^{[9-11]}$, which are serious hazards. The main treatment

44 for hyperuricemia is using uric acid-lowering agents. The relationship between uric acid lowering 
45 and renal disease has received extensive attention. Some randomized controlled trial (RCT)

46 studies have shown that using uric acid-lowering agents can improve kidney disease ${ }^{[12-18]}$.

47 Furthermore, animal experiment results show that using uric acid-lowering agents improves

48 fibrosis through mechanisms that include improving oxidative stress ${ }^{[19-22]}$ and reducing the

49 expression of inflammatory factors ${ }^{[23]}$. However, other RCT studies have not found a relationship

50 between uric acid-lowering agents and chronic kidney disease (CKD) ${ }^{[24-29]}$, indicating that there

51 may be other mechanism besides uric acid-lowering agents to improve renal disease. Zhou, H. B,

52 et al ${ }^{[8]}$ found that in patients with acute heart failure, worse renal function was significantly more

53 common in patients experiencing increased uric acid (UA). Takayuki Tsuji, et al ${ }^{[30]}$ reported a

54 retrospective observational study and found that serum uric acid reduction might have beneficial

55 effects on CKD progression in CKD patients with hyperuricemia. Studies have reported the

56 relationship between uric acid change and some chronic diseases, but no study exploring the

57 association between uric acid lowering and the incidence of renal disease with no drug

58 application has been reported.

59 This study is aimed to explore the association between automatic uric acid lowering and renal

60 function progression in community population with a longitudinal study. The results may provide

61 recommendations for uric acid management.

\section{Materials \& Methods}

\section{Study population and design}

65 Participants were recruited from a community-based cohort study in Guangzhou, China, which

66 was designed as a single-center prospective observational study to evaluate chronic diseases in

67 the Chinese population. During the recruitment period, local permanent residents were invited to

68 participate in a screening examination for diabetes, from June to December 2012. The inclusion 
69 criteria included the following: (1) age $\geq 40$ and $\leq 75$ years old and (2) voluntarily participated in a

70 continuous follow-up for 4 years and voluntarily cooperated with follow-up requirements. The

71 exclusion criteria included the following: (1) a history of cancer or autoimmune diseases, (2)

72 acute diabetic complications, such as acidosis, (3) moderate to severe liver or kidney dysfunction,

73 that is, alanine aminotransferase (ALT) /aspartate aminotransferase (AST) $>2.5$ times the upper

74 limit of the normal range or creatinine clearance $<25 \mathrm{~mL} / \mathrm{min}$, (4) other condition or major

75 systemic disease that interferes with trial participation or evaluation, and (5) usage of uric acid

76 lowering drugs in recent one year. A total of 2876 community residents were enrolled in this

77 study. There were 402 cases of missing data in the baseline questionnaire, with a response rate of

78 86.02\%. In 2016, participants returned for a 4-year follow-up survey, and 2334 participants

79 completed the baseline and follow-up surveys (follow-up rate was $78.0 \%$ ). In this study,

80 excluding subjects unqualified by questionnaire $(n=7)$, without matching data in $2012(n=309)$,

81 missing all blood results in $2012(\mathrm{n}=80)$ or missing serum uric acid or eGFR $(\mathrm{n}=45), 1489$

82 eligible individuals were included in the final analyses of the association between uric acid

83 lowering and renal function progression. The flow chart of inclusion and exclusion of subjects in

84 this study is shown in figure 1.

85 The subjects was devided into four groups according to the interquartile interval of change of

86 serum uric acid between baseline and 2016, including Quartile 1 (<-91.00 mol/L) with

87 significantly reduced serum uric acid, Quartile 2 (-91.00--36.68 mol/L) with slightly reduced

88 serum uric acid (36.68-91.00 mol/L) and Quartile 3 with basically stable serum uric acid

89 (decreased $\leq 36.68 \mathrm{~mol} / \mathrm{L}$ or increased $<12.34 \mathrm{~mol} / \mathrm{L})$, and Quartile $4(\geq 12.34 \mathrm{~mol} / \mathrm{L})$ with

90 increased serum uric acid.

91 All participants signed informed consent forms before data collection. The study conformed to

92 the principles of the Helsinki Declaration II and was approved by the ethics committee of Sun

93 Yat-sen Memorial Hospital affiliated with Sun Yat-sen University (2014[33]). 


\section{Clinical and biochemical measurements}

95 Venous blood samples were collected for laboratory tests after an overnight fasting for at least 10

96 hours. Blood and urine samples were stored and tested according to the requirements of the

97 instructions. Fasting plasma glucose (FPG), 2-hour plasma glucose (2hPG), fasting serum insulin

98 (INS), triglycerides (TG), total cholesterol (TC), high-density lipoprotein cholesterol (HDL-C),

99 low-density lipoprotein cholesterol (LDL-C), serum creatinine (Scr), AST and ALT, total

100 bilirubin (TBIL), blood urea nitrogen (BUN), uric acid (UA), and urine creatinine (Ucr) were

101 measured using an autoanalyzer (Beckman AU5831 Biochemical Autoanalyser, Beckman, USA).

102 Urine microalbumin (UALB) was measured using an automatic protein analyzer (BN II System,

103 Siemens ag, Germany). Glycated hemoglobin A1c (HbA1c) was assessed by high-performance

104 liquid chromatography (VARIANT II TURBO, BIO-RAD, USA). Thyroid-stimulating hormone

105 (TSH), thyroid peroxidase antibody (TPOAb), free thyroxine (FT4), and INS were assessed using

106 an automatic chemiluminescence immunoanalyzer (ADVIA Centaur XP, Siemens ag, Germany).

107 All laboratory tests and quality controls were conducted in the laboratory of clinical laboratory 108 and endocrinology laboratory of Sun Yat-sen Memorial Hospital, Sun Yat-sen University. The

109 insulin resistance index (homeostasis model assessment of insulin resistance, HOMA-IR) was

110 calculated as fasting insulin $(\mu \mathrm{IU} / \mathrm{mL}) \times$ fasting glucose $(\mathrm{mmol} / \mathrm{L}) / 22.5$. EGFR $(\mathrm{mL} /(\mathrm{min} \times 1.73$

$\left.111 \mathrm{~m}^{2}\right)$ ) was calculated using a formula of $186 \times(\operatorname{Scr}(\mu \mathrm{mol} / \mathrm{L}) \times 0.011)-1.154 \times$ age -0.203

$112(\times 0.742$ female $) \times 1.233$.

\section{Diagnostic criteria}

114 Diabetes was diagnosed as FPG $\geq 7.0 \mathrm{mmol} / \mathrm{L}$, or $2-\mathrm{h} \mathrm{PG} \geq 11.1 \mathrm{mmol} / \mathrm{L}$ during an oral glucose

115 tolerance test (OGTT), or HbA1c $\geq 6.5 \%$. Prediabetes was diagnosed as FPG $5.6 \mathrm{mmol} / \mathrm{L}$ to 6.9

$116 \mathrm{mmol} / \mathrm{L}$, or $2-\mathrm{h}$ PG $7.8 \mathrm{mmol} / \mathrm{L}$ to $11.0 \mathrm{mmol} / \mathrm{L}$ during $75 \mathrm{~g}$ OGTT, or HbA1c $5.7-6.4 \%$

117 according to the 2019 American Diabetes Association (ADA) diagnostic criteria ${ }^{[31]}$. 
118 Hyperuricemia was diagnosed as serum uric acid $>420 \mu \mathrm{mol} / \mathrm{L}$, according to the 2017

119 multidisciplinary expert consensus on the diagnosis and treatment of hyperuricemia-related

120 diseases in China ${ }^{[32]}$. Hypertension was diagnosed as systolic blood pressure (SBP) $\geq 140 \mathrm{mmHg}$

121 or diastolic blood pressure $(\mathrm{DBP}) \geq 90 \mathrm{mmHg}$ according to the $2018 \mathrm{ESH} / \mathrm{ESC}$ guidelines

122 recommendations ${ }^{[33]}$ or taking hypotensive drugs regardless of blood pressure.

\section{Definition of renal function progression}

124 Renal function decline was defined as eGFR decreased more than 10\% from baseline in 2016.

125 Renal function improvement was defined as eGFR increased more than $10 \%$ from baseline in $1262016^{[34,35]}$.

\section{Statistical analysis}

128 All continuous variables are presented as means \pm standard deviation except for skewed

129 variables, which are presented as medians (interquartile ranges). Continuous variables with

130 normal distribution, $t$ test or variance analysis were used to compare differences between groups.

131 Wilcoxon or Kruskal-Wallis tests were used for comparisons between every two groups for 132 variables with nonnormal distribution. The classified data are expressed as frequency 133 (percentage), and the chi-square test was used for comparisons between groups. The unadjusted

134 and multivariate-adjusted Cox regression analysis was used for the assessment of risk ratios of 135 different groups and the calculation of HR and 95\% CI. Relationships between different changes

136 in uric acid and renal function improvement were also explored in subgroups stratified by gender 137 (male, female), age $(<60 / \geq 60$ years), glucose tolerance status (normal glucose tolerance, 138 prediabetes or diabetes), high blood pressure (yes/no), hyperuricemia (yes/no), and BMI $139\left(<24 / \geq 24 \mathrm{~kg} / \mathrm{m}^{2}\right)$. Tests for interaction were performed, simultaneously including each strata 140 factor, the quartiles of change of serum uric acid and the respective interaction terms in the 141 models. SPSS 22.0 statistical software was used for analysis. All statistical tests were two-sided, 142 and $\mathrm{P}<0.05$ was considered statistically significant. 


\section{Results}

\section{Clinical characteristics of the study population}

146 A total of 1523 subjects were included in this study, with a median age of 57.26 (53.39 - 61.62)

147 years and a median uric acid level of 394.26 (334.97 - 462.82) $\mu \mathrm{mol} / \mathrm{L}$ at baseline. The 148 prevalence of diabetes and prediabetes in baseline were respectively $20.9 \%$ and $56.5 \%$. The 149 baseline characteristics of different groups are shown in table 1. Compared to quartile 2 and

150 quartile 3, the proportions of male, waist-to-hip ratios and weights of quartile 1 and quartile 4 151 were higher $(\mathrm{P}<0.05)$. In quartile 2 , TC, TG, ALT and UA levels were higher $(\mathrm{P}<0.05)$. The 152 eGFR was not significantly different between groups $(\mathrm{P}>0.05)$.

\section{Association of uric acid lowering with renal function progression}

154 By 2016, a total of 421 subjects had renal function decline, with an incidence of 28.3\%. Quartile 1551 represented lower quarter. The incidence of renal function decline in different groups is shown 156 in figure 2A. As the quartile increased, the incidence increased from $20.6 \%$ to $39.6 \%(P<0.05)$.

157 By 2016, 267 subjects with renal dysfunction had improved renal function, and the incidence of 158 renal function improvement was $17.9 \%$. The incidence rate of renal function improvement in the 159 population with renal dysfunction in different groups is shown in figure $2 \mathrm{~B}$. As the quartile 160 increased, the incidence decreased from $30.7 \%$ to $10.0 \%(\mathrm{P}<0.05)$.

161 As shown in table 2, the risk of renal function decline was lower in quartile 1, quartile 2 and 162 quartile 3 compared with quartile 4. After adjusting for gender, age, dietary exercise intervention, 163 Waist-to-Hip Ratio, BMI, pulse pressure variation, 2hPG variation, AST variation, ALT 164 variation, TC variation, the risks of renal function decline in quartile 1 , quartile 2 and quartile 3 165 were 64\% $(\mathrm{HR}=0.64,95 \% \mathrm{CI}: 0.49-0.85), 65 \%(\mathrm{HR}=0.65,95 \% \mathrm{CI}: 0.50-0.84)$ and $75 \%$ $166(\mathrm{HR}=0.75,95 \% \mathrm{CI}: 0.58$ - 0.96) $(\mathrm{P}<0.05)$, respectively, compared with quartile 4. 
167 As shown in table 3, after adjusting for gender, age, dietary exercise intervention, BMI, UA,

168 HOMA-IR, HDL-C, HbA1c variation, ALT variation , AST varaition., the incidence of renal 169 function improvement in quartile 1 and quartile 2 was 2.27 times (HR = 2.27, 95\% CI: 1.45 -

$1703.57)$ and 1.78 times higher ( $\mathrm{HR}=1.78,95 \% \mathrm{CI}: 1.17-2.69)$, respectively, than that in quartile 4

$171(\mathrm{P}<0.05)$.

172 The association between serum uric acid lowering and progression of renal function was further

173 explored in subgroups. The results are shown in figure 3 and figure 4 . The result for each 174 subgroup was almost consistent with the results for the general population.

176 Discussion

177 In this study, we found that uric acid lowering was significantly associated with decreased risk of 178 renal function decline and increased incidence of renal function improvement. The association 179 remains after analysis in subgroups.

180 The incidence of renal function decline in an RCT study ${ }^{[12]}$ with 108 subjects was $54.4 \%$, which 181 was higher than our study, because of different characteristics of population. Our research 182 subjects were significantly larger so that the population in our study may be more representative. 183 In a cohort study ${ }^{[8]}$, the incidence was $11.6 \%$ which was similar to our study.

184 Hyperuricemia is associated with many chronic diseases. For example, hypertension ${ }^{[2,3]}$, diabetes $185^{[4,5]}$ and cardiovascular disease ${ }^{[6-8]}$ interact with hyperuricemia. The relationship between uric acid 186 and renal function has received widespread attention in recent years. Sircar D, et al ${ }^{[12]}$, based on 187 an RCT study conducted in eastern India with 6 months of follow-up in 108 participants, showed 188 that the febuxostat can delay progress in chronic kidney disease in patients with CKD and HUA 189 with uric acid lowering, which is consistent with our findings. However, febuxostat may 190 influence renal function change by other mechanisms at the same time, which may overestimate 
191 the association between uric acid lowering and renal function. Zhou, H. B, et al ${ }^{[8]}$ found that in

192 patients with acute heart failure, worse renal function was significantly more common in patients

193 experiencing increased UA based on a cohort study in 535 participants. Takayuki Tsuji, et al [30]

194 reported a retrospective observational study with 86 participants and found that serum uric acid

195 reduction might have beneficial effects on CKD progression of CKD patients with

196 hyperuricemia. These results were consistent with our findings, but these studies were based on a

197 population with disease or with a small sample size, limiting the exploration of the relationship

198 between uric acid and renal disease. Moreover, the baseline uric acid levels in the different

199 groups in these studies were significantly different, which may misestimate the results, as

200 hyperuricemia was found be an independent predictor for the development of newly diagnosed

$201 \mathrm{CKD}^{[10]}$. Our study excluded subjects with usage of uric acid-lowering agents, avoiding the

202 influence of other factors, suggesting the exact association between uric acid lowering and renal

203 function. Baseline serum uric acid levels were adjusted when exploring the relationship between

204 uric acid lowering and changes in renal function in our study. Moreover, our longitudinal study

205 has followed up for four years.

206 Our study found that uric acid lowering was beneficially associated with changes in renal

207 function. In subgroup analysis, even for the subjects without hyperuricemia, blood uric acid

208 lowering is beneficial for renal function change. In the study, about $70 \%$ subjects had lower uric

209 acid in 2016 than in 2012. Although we exclude subjects with uric acid lowering agents in 2012,

210 we only assessed uric acid lowering therapies in first visit which could not avoid latter usage.

211 However, the prevalence of hyperuricemia in Chinese was $13.3 \%^{[1]}$, and the ratio of awareness

212 and treatment were $0.9 \%{ }^{[36]}$ and $50 \%{ }^{[37]}$, respectively. With the low prevalence, awareness and

213 treatment rate of hyperuricemia. The rate of usage of uric acid lowering agents in our study in

2142016 can be extremely low. The lowering of uric acid in our study was not due to the usage of

215 uric acid lowering agents. The prevalence of diabetes and prediabetes in baseline was 
216 respectively $20.9 \%$ and $56.5 \%$. The proportion of abnormal glucose metabolism in our study was

217 in high level, and health consciousness of subjects who participated in the study could be high.

218 Diet and exercise may play an important role in the uric acid lowering. Most uric acid-lowering

219 agents should not be used when severe renal dysfunction occurs, such as febuxostat and

220 benzbromarone, and the risk of adverse reactions of allopurinol increased in patients with renal

221 insufficiency ${ }^{[32]}$. Considering the possible adverse reactions of uric acid-lowering agents, diet

222 adjustment, improving life habits and avoiding drugs that increase serum uric acid levels are

223 recommended first measures. It is also worth noting that early uric acid-lowering management

224 can avoid the dilemma of uric acid-lowering therapy in severe renal insufficiency.

225 There can be several mechanisms through which uric acid influences renal function. First, uric

226 acid is filtered through the kidney, and increased serum uric acid may lead to the formation of

227 uric acid crystals in the kidney ${ }^{[38]}$, which cause injured renal epithelial cells, forming an acute

228 inflammatory response ${ }^{[39]}$ and renal stones. Second, elevated uric acid may lead to renal vascular

229 lesions by activating the renin-angiotensin-aldosterone system ${ }^{[40,41]}$. Third, hyperuricemia can

230 lead to anterior glomerular vascular lesions, affect the self-regulation of the afferent arterioles,

231 and lead to high pressure in the glomerulus, while the thickening of the blood vessel wall can

232 lead to vascular obstruction and renal hypoperfusion, and the hypoxia can lead to

233 tubulointerstitial inflammation and fibrosis ${ }^{[40,41]}$. Decreased serum uric acid may improve renal

234 function by blocking or improving the above mechanisms.

235 There are some limitations to be considered. First, the subjects in this study were Chinese; the

236 adaptability and application of the results to foreign areas is limited, and bias cannot be avoided,

237 even in domestic populations because of China's vast territory. Second, the population was

238 predominantly female because the community population in this study was 40 years or older,

239 which is predominantly female. Additionally, this study was limited to residents over 40 years

240 old in the Guangzhou community; thus, the conclusions in this study have limited application in a 
241 young population. Third, the follow-up rate in this study was $78.0 \%$, which is low, but similar to

242 other large epidemiological studies ${ }^{[42]}$. Fourth, we only assessed uric acid lowering therapies in

243 first visit which could not avoid latter usage of uric acid lowering drugs during the next four year.

244 However, as far as we known, the prevalence of hyperuricemia in Chinese was $13.3 \%{ }^{[1]}$, and the

245 ratio of awareness and treatment were $0.9 \%{ }^{[36]}$ and $50 \%{ }^{[37]}$, respectively. Moreover, national

246 Health and Nutrition Examination Survey in US showed that prevalence of hyperuricemia was

247 about $20 \%$, while the prevalence of uric acid lowering drugs using among patients with gout was

$24833 \%{ }^{[43]}$. Therefore, we can conclude that the proportion of participants receiving treatment of

249 uric acid lowering drugs is very low, which prompt that there may be only a small part of

250 participants receving uric acid lowing treatment and may not have a great impact on this result.

251 Fifth, we did not collect diabetes disease duration in the study. However, there was no

252 stasistically significant difference in FPG, 2hPG and HbA1c between four study groups. Studies

253 reported that hyperuricemia seems to be an independent risk factor for the development of

254 incident CKD even adjusted diabetes duration ${ }^{[44,45]}$. Therefore, we suggested that the missing

255 information of diabetes disease duration may not influence the relationship between uric acid and

256 CKD. Besides, relationships between changes in uric acid and renal function were also explored

257 in subgroups stratified by glucose tolerance status (normal glucose tolerance, prediabetes or

258 diabetes) with consistent results for the general population. However, the study will be more

259 rigorous if more detailed information of continuous uric acid lowering therapies assessment and 260 diabetes disease duration was collected.

262 Conclusions

263 Uric acid lowering is associated with changes in renal function. The association between uric acid

264 lowering and change in renal function is also found in the non-hyperuricemia population. The 
265 study provides support for clinical strategy and community health management. Uric acid266 lowering therapy should be timely and appropriate and ought to be a part of the comprehensive 267 management of chronic renal disease.

268

269 References

270 [1] Liu R, Han C, Wu D, Xia X, Gu J, Guan H, Shan Z, Teng W. Prevalence of 271 Hyperuricemia and Gout in Mainland China from 2000 to 2014: A Systematic Review and Meta272 Analysis. Biomed Res Int, 2015, 2015: 762820.

273 [2] Wei F, Sun N, Cai C, Feng S, Tian J, Shi W, Xu W, Wang Y, Yang X, Li WD.

274 Associations between serum uric acid and the incidence of hypertension: a Chinese senior 275 dynamic cohort study. J Transl Med, 2016, 14(1): 110.

276 [3] Kuwabara M, Niwa K, Nishi Y, Mizuno A, Asano T, Masuda K, Komatsu I, Yamazoe M, 277 Takahashi O, Hisatome I. Relationship between serum uric acid levels and hypertension among 278 Japanese individuals not treated for hyperuricemia and hypertension. Hypertens Res, 2014, 37(8): $279785-789$.

280 [4] Liu J, Tao L, Zhao Z, Mu Y, Zou D, Zhang J, Guo X. Two-Year Changes in 281 Hyperuricemia and Risk of Diabetes: A Five-Year Prospective Cohort Study. J Diabetes Res, 282 2018, 2018: 6905720.

283 [5] Mortada I. Hyperuricemia, Type 2 Diabetes Mellitus, and Hypertension: an Emerging 284 Association. Curr Hypertens Rep, 2017, 19(9): 69.

285 [6] Li M, Hu X, Fan Y, Li K, Zhang X, Hou W, Tang Z. Hyperuricemia and the risk for 286 coronary heart disease morbidity and mortality a systematic review and dose-response meta287 analysis. Sci Rep, 2016, 6: 19520. 
288 [7] Capuano V, Marchese F, Capuano R, Torre S, Iannone AG, Capuano E, Lamaida N, 289 Sonderegger M, Capuano E. Hyperuricemia as an independent risk factor for major 290 cardiovascular events: a 10-year cohort study from Southern Italy. J Cardiovasc Med 291 (Hagerstown), 2017, 18(3): 159-164.

292 [8] Zhou HB, Xu TY, Liu SR, Bai YJ, Huang XF, Zhan Q, Zeng QC, Xu DL. Association of 293 serum uric acid change with mortality, renal function and diuretic dose administered in treatment 294 of acute heart failure. Nutr Metab Cardiovasc Dis, 2019.

295 [9] Toda A, Ishizaka Y, Tani M, Yamakado M. Hyperuricemia is a significant risk factor for 296 the onset of chronic kidney disease. Nephron Clin Pract, 2014, 126(1): 33-38.

297 [10] Li L, Yang C, Zhao Y, Zeng X, Liu F, Fu P. Is hyperuricemia an independent risk factor 298 for new-onset chronic kidney disease?: A systematic review and meta-analysis based on 299 observational cohort studies. BMC Nephrol, 2014, 15: 122.

300 [11] Maloberti A, Maggioni S, Occhi L, Triglione N, Panzeri F, Nava S, Signorini S, Falbo R, 301 Casati M, Grassi G, Giannattasio C. Sex-related relationships between uric acid and target organ 302 damage in hypertension. J Clin Hypertens (Greenwich), 2018, 20(1): 193-200.

303 [12] Sircar D, Chatterjee S, Waikhom R, Golay V, Raychaudhury A, Chatterjee S, Pandey R. 304 Efficacy of Febuxostat for Slowing the GFR Decline in Patients With CKD and Asymptomatic 305 Hyperuricemia: A 6-Month, Double-Blind, Randomized, Placebo-Controlled Trial. Am J Kidney 306 Dis, 2015, 66(6): 945-950.

307 [13] Liu P, Wang H, Zhang F, Chen Y, Wang D, Wang Y. The Effects of Allopurinol on the 308 Carotid Intima-media Thickness in Patients with Type 2 Diabetes and Asymptomatic 309 Hyperuricemia: A Three-year Randomized Parallel-controlled Study. Intern Med, 2015, 54(17): $310 \quad 2129-2137$. 
311 [14] Chen JH, Lan JL, Cheng CF, Liang WM, Lin HY, Tsay GJ, Yeh WT, Pan WH. Effect of

312 Urate-Lowering Therapy on All-Cause and Cardiovascular Mortality in Hyperuricemic Patients

313 without Gout: A Case-Matched Cohort Study. PLoS One, 2015, 10(12): e0145193.

314 [15] Rekhraj S, Gandy SJ, Szwejkowski BR, Nadir MA, Noman A, Houston JG, Lang CC,

315 George J, Struthers AD. High-dose allopurinol reduces left ventricular mass in patients with 316 ischemic heart disease. J Am Coll Cardiol, 2013, 61(9): 926-932.

317 [16] Soletsky B, Feig DI. Uric acid reduction rectifies prehypertension in obese adolescents. 318 Hypertension, 2012, 60(5): 1148-1156.

319 [17] Noman A, Ang DS, Ogston S, Lang CC, Struthers AD. Effect of high-dose allopurinol on 320 exercise in patients with chronic stable angina: a randomised, placebo controlled crossover trial. 321 Lancet, 2010, 375(9732): 2161-2167.

322 [18] Kanji T, Gandhi M, Clase CM, Yang R. Urate lowering therapy to improve renal 323 outcomes in patients with chronic kidney disease: systematic review and meta-analysis. BMC 324 Nephrol, 2015, 16: 58.

325 [19] Xu X, Hu X, Lu Z, Zhang P, Zhao L, Wessale JL, Bache RJ, Chen Y. Xanthine oxidase 326 inhibition with febuxostat attenuates systolic overload-induced left ventricular hypertrophy and 327 dysfunction in mice. J Card Fail, 2008, 14(9): 746-753.

328 [20] George J, Carr E, Davies J, Belch JJ, Struthers A. High-dose allopurinol improves 329 endothelial function by profoundly reducing vascular oxidative stress and not by lowering uric 330 acid. Circulation, 2006, 114(23): 2508-2516.

331 [21] Kittleson MM, Hare JM. Xanthine oxidase inhibitors: an emerging class of drugs for heart 332 failure. Eur Heart J, 2005, 26(15): 1458-1460.

333 [22] Engberding N, Spiekermann S, Schaefer A, Heineke A, Wiencke A, Muller M, Fuchs M, 334 Hilfiker-Kleiner D, Hornig B, Drexler H, Landmesser U. Allopurinol attenuates left ventricular 
335 remodeling and dysfunction after experimental myocardial infarction: a new action for an old 336 drug? Circulation, 2004, 110(15): 2175-2179.

337 [23] Omori H, Kawada N, Inoue K, Ueda Y, Yamamoto R, Matsui I, Kaimori J, Takabatake Y,

338 Moriyama T, Isaka Y, Rakugi H. Use of xanthine oxidase inhibitor febuxostat inhibits renal 339 interstitial inflammation and fibrosis in unilateral ureteral obstructive nephropathy. Clin Exp 340 Nephrol, 2012, 16(4): 549-556.

341 [24] Kimura K, Hosoya T, Uchida S, Inaba M, Makino H, Maruyama S, Ito S, Yamamoto T, 342 Tomino Y, Ohno I, Shibagaki Y, Iimuro S, Imai N, Kuwabara M, Hayakawa H, Ohtsu H, Ohashi 343 Y. Febuxostat Therapy for Patients With Stage 3 CKD and Asymptomatic Hyperuricemia: A 344 Randomized Trial. Am J Kidney Dis, 2018, 72(6): 798-810.

345 [25] Zhang J, Dierckx R, Mohee K, Clark AL, Cleland JG. Xanthine oxidase inhibition for the 346 treatment of cardiovascular disease: an updated systematic review and meta-analysis. ESC Heart 347 Fail, 2017, 4(1): 40-45.

348 [26] McMullan CJ, Borgi L, Fisher N, Curhan G, Forman J. Effect of Uric Acid Lowering on 349 Renin-Angiotensin-System Activation and Ambulatory BP: A Randomized Controlled Trial. Clin 350 J Am Soc Nephrol, 2017, 12(5): 807-816.

351 [27] Givertz MM, Anstrom KJ, Redfield MM, Deswal A, Haddad H, Butler J, Tang WH, 352 Dunlap ME, LeWinter MM, Mann DL, Felker GM, O'Connor CM, Goldsmith SR, Ofili EO, 353 Saltzberg MT, Margulies KB, Cappola TP, Konstam MA, Semigran MJ, McNulty SE, Lee KL, 354 Shah MR, Hernandez AF. Effects of Xanthine Oxidase Inhibition in Hyperuricemic Heart Failure 355 Patients: The Xanthine Oxidase Inhibition for Hyperuricemic Heart Failure Patients (EXACT356 HF) Study. Circulation, 2015, 131(20): 1763-1771.

357 [28] Ogino K, Kato M, Furuse Y, Kinugasa Y, Ishida K, Osaki S, Kinugawa T, Igawa O, 358 Hisatome I, Shigemasa C, Anker SD, Doehner W. Uric acid-lowering treatment with 
359 benzbromarone in patients with heart failure: a double-blind placebo-controlled crossover 360 preliminary study. Circ Heart Fail, 2010, 3(1): 73-81.

361 [29] Zhang T, Pope JE. Cardiovascular effects of urate-lowering therapies in patients with

362 chronic gout: a systematic review and meta-analysis. Rheumatology (Oxford), 2017, 56(7): 11443631153.

364 [30] Tsuji T, Ohishi K, Takeda A, Goto D, Sato T, Ohashi N, Fujigaki Y, Kato A, Yasuda H.

365 The impact of serum uric acid reduction on renal function and blood pressure in chronic kidney 366 disease patients with hyperuricemia. Clin Exp Nephrol, 2018, 22(6): 1300-1308.

367 [31] Classification and Diagnosis of Diabetes: Standards of Medical Care in Diabetes-2019. 368 Diabetes Care, 2019, 42(Suppl 1): S13-s28.

369 [32] Mei Changlin, Ge Junbo, Zou Hejian, Gao Xin. 2017 Chinese multi-disciplinary 370 consensus on the diagnosis and treatment of hyperuricemia and its related diseases. Chin J Intern 371 Med, March 2017, 56(3): 235-248.

372 [33] Cuspidi C, Tadic M, Grassi G, Mancia G. Treatment of hypertension: The ESH/ESC 373 guidelines recommendations. Pharmacol Res, 2018, 128: 315-321.

374 [34] Ishihara H, Kondo T, Fukuda H, Yoshida K, Omae K, Takagi T, Iizuka J, Kobayashi H, 375 Tanabe K. Evaluation of renal function change during first-line tyrosine kinase inhibitor therapy 376 for metastatic renal cell carcinoma. Jpn J Clin Oncol, 2017, 47(12): 1175-1181.

377 [35] Matsushita K, Chen J, Sang Y, Ballew SH, Shimazaki R, Fukagawa M, Imai E, Coresh J, 378 Hishida A. Risk of end-stage renal disease in Japanese patients with chronic kidney disease 379 increases proportionately to decline in estimated glomerular filtration rate. Kidney Int, 2016, 380 90(5): 1109-1114.

381 [36] Xue C, Ye XD, Li W, Peng Q, Ding HY, Zhang YH, He DF, Bai X, Huang Y, Song YS, 382 Pang L, Liao YH. Prevalence of chronic kidney disease in Jing adults in China: a village-based 383 study. Clin Nephrol, 2013, 79(1): 50-56. 
384 [37] Yamamoto Y, Matsubara K, Igawa G, Kaetsu Y, Sugihara S, Matsuura T, Ando F,

385 Sonoyama K, Hamada T, Ogino K, Igawa O, Shigemasa C, Hisatome I. Status of uric acid 386 management in hypertensive subjects. Hypertens Res, 2007, 30(6): 549-554.

387 [38] Koka RM, Huang E, Lieske JC. Adhesion of uric acid crystals to the surface of renal 388 epithelial cells. Am J Physiol Renal Physiol, 2000, 278(6): F989-998.

389 [39] Diwan V, Mistry A, Gobe G, Brown L. Adenine-induced chronic kidney and 390 cardiovascular damage in rats. J Pharmacol Toxicol Methods, 2013, 68(2): 197-207.

391 [40] Sanchez-Lozada LG, Tapia E, Santamaria J, Avila-Casado C, Soto V, Nepomuceno T, 392 Rodriguez-Iturbe B, Johnson RJ, Herrera-Acosta J. Mild hyperuricemia induces vasoconstriction 393 and maintains glomerular hypertension in normal and remnant kidney rats. Kidney Int, 2005, 394 67(1): 237-247.

395 [41] Kosugi T, Nakayama T, Heinig M, Zhang L, Yuzawa Y, Sanchez-Lozada LG, Roncal C, 396 Johnson RJ, Nakagawa T. Effect of lowering uric acid on renal disease in the type 2 diabetic 397 db/db mice. Am J Physiol Renal Physiol, 2009, 297(2): F481-488.

398 [42] Hägglund G, Pettersson K, Czuba T, Persson-Bunke M, Rodby-Bousquet E. Incidence of 399 scoliosis in cerebral palsy. Acta Orthop, 2018, 89(4): 443-447.

400 [43] Chen-Xu M, Yokose C, Rai SK, Pillinger MH, Choi HK. Contemporary Prevalence of 401 Gout and Hyperuricemia in the United States and Decadal Trends: The National Health and 402 Nutrition Examination Survey 2007-2016. Arthritis Rheumatol, 2019.

403 [44] Zoppini G, Targher G, Chonchol M, Ortalda V, Abaterusso C, Pichiri I, Negri C, Bonora

404 E. Serum uric acid levels and incident chronic kidney disease in patients with type 2 diabetes and 405 preserved kidney function. Diabetes Care, 2012, 35(1): 99-104.

406 [45] Yan D, Tu Y, Jiang F, Wang J, Zhang R, Sun X, Wang T, Wang S, Bao Y, Hu C, Jia W. 407 Uric Acid is independently associated with diabetic kidney disease: a cross-sectional study in a 408 Chinese population. PLoS One, 2015, 10(6): e0129797. 


\section{Table 1 (on next page)}

Baseline characteristics of study population by change of serum uric acid levels 
Table 1. Baseline characteristics of study population by change of serum uric acid levels

\begin{tabular}{|c|c|c|c|c|c|}
\hline Types & $\begin{array}{l}\text { Quartile1 } \\
(<-91.00) \\
(\mathrm{n}=381)\end{array}$ & $\begin{array}{c}\text { Quartile2 } \\
(-91.00--36.68) \\
(\mathrm{n}=381)\end{array}$ & $\begin{array}{c}\text { Quartile3 } \\
(-36.68-12.34) \\
(\mathrm{n}=381)\end{array}$ & $\begin{array}{l}\text { Quartile4 } \\
(\geq 12.34) \\
(\mathrm{n}=380)\end{array}$ & $\mathrm{P}$ \\
\hline Age (Years) & $57.00(52.93-61.32)$ & $57.53(53.69-61.79)$ & $57.12(53.57-61.24)$ & $57.65(53.37-62.35)$ & 0.335 \\
\hline Sex & & & & & 0.017 \\
\hline Male & $110(28.9)^{\#}$ & $92(24.1)^{*}$ & $85(22.3)^{*}$ & $119(31.4)$ & \\
\hline Female & $271(71.1)$ & $289(75.9)$ & $296(77.7)$ & $260(68.6)$ & \\
\hline Smoking & & & & & 0.485 \\
\hline Yes & $31(12.0)$ & $28(10.7)$ & $25(9.3)$ & $37(13.4)$ & \\
\hline No & $227(88.0)$ & $233(89.3)$ & $244(90.7)$ & $240(86.6)$ & \\
\hline Drinking & & & & & 0.806 \\
\hline Yes & $131(42.0)$ & $121(38.5)$ & $122(38.7)$ & $121(39.7)$ & \\
\hline No & $181(58.0)$ & $193(61.5)$ & $190(61.3)$ & $184(60.3)$ & \\
\hline Family History of DM & & & & & 0.448 \\
\hline Yes & $89(24.0)$ & $78(21.0)$ & $71(19.2)$ & $83(22.4)$ & \\
\hline No & $282(76.0)$ & $293(79.0)$ & $298(80.8)$ & $288(77.6)$ & \\
\hline FPG (mmol/L) & $5.87(5.31-6.50)$ & $5.81(5.37-6.38)$ & $5.73(5.26-6.28)$ & $5.80(5.36-6.39)$ & 0.302 \\
\hline 2hPG (mmol/L) & $7.30(5.80-9.58)$ & $7.30(5.72-9.31)$ & $6.90(5.66-9.00)$ & $7.30(5.90-9.70)$ & 0.201 \\
\hline HbA1c (\%) & $5.70(5.30-6.00)$ & $5.60(5.40-6.00)$ & $5.60(5.30-5.90)$ & $5.60(5.30-5.90)$ & 0.177 \\
\hline HOMA-IR & $2.83(2.01-4.05)$ & $2.79(1.96-3.97)$ & $2.74(1.80-3.91)$ & $2.86(2.03-4.15)$ & 0.356 \\
\hline $\mathrm{P}(/ \mathrm{min})$ & $80.00(73.00-88.00)$ & $80.00(73.00-89.50)$ & $80.00(74.00-88.00)$ & $80.00(72.25-89.00)$ & 0.892 \\
\hline SBP (mmHg) & $132.00(121.00-145.00)$ & $133.00(121.00-146.00)$ & $133.00(122.00-146.00)$ & $133.00(123.00-144.00)$ & 0.972 \\
\hline $\mathrm{DBP}(\mathrm{mmHg})$ & $76.00(68.00-83.00)$ & $75.00(69.00-82.00)$ & $76.00(69.00-82.00)$ & $76.00(69.00-83.75)$ & 0.596 \\
\hline Pulse pressure $(\mathrm{mmHg})$ & $56.00(48.00-67.00)$ & $58.00(49.00-67.00)$ & $58.00(48.00-67.00)$ & $56.00(48.00-65.00)$ & 0.608 \\
\hline \multicolumn{6}{|c|}{ Continued to Table 1} \\
\hline Types & $\begin{array}{l}\text { Quartile1 } \\
(<-91.00)\end{array}$ & $\begin{array}{c}\text { Quartile2 } \\
(-91.00--36.68)\end{array}$ & $\begin{array}{c}\text { Quartile3 } \\
(-36.68-12.34)\end{array}$ & $\begin{array}{l}\text { Quartile4 } \\
(\geq 12.34)\end{array}$ & $\mathrm{P}$ \\
\hline
\end{tabular}




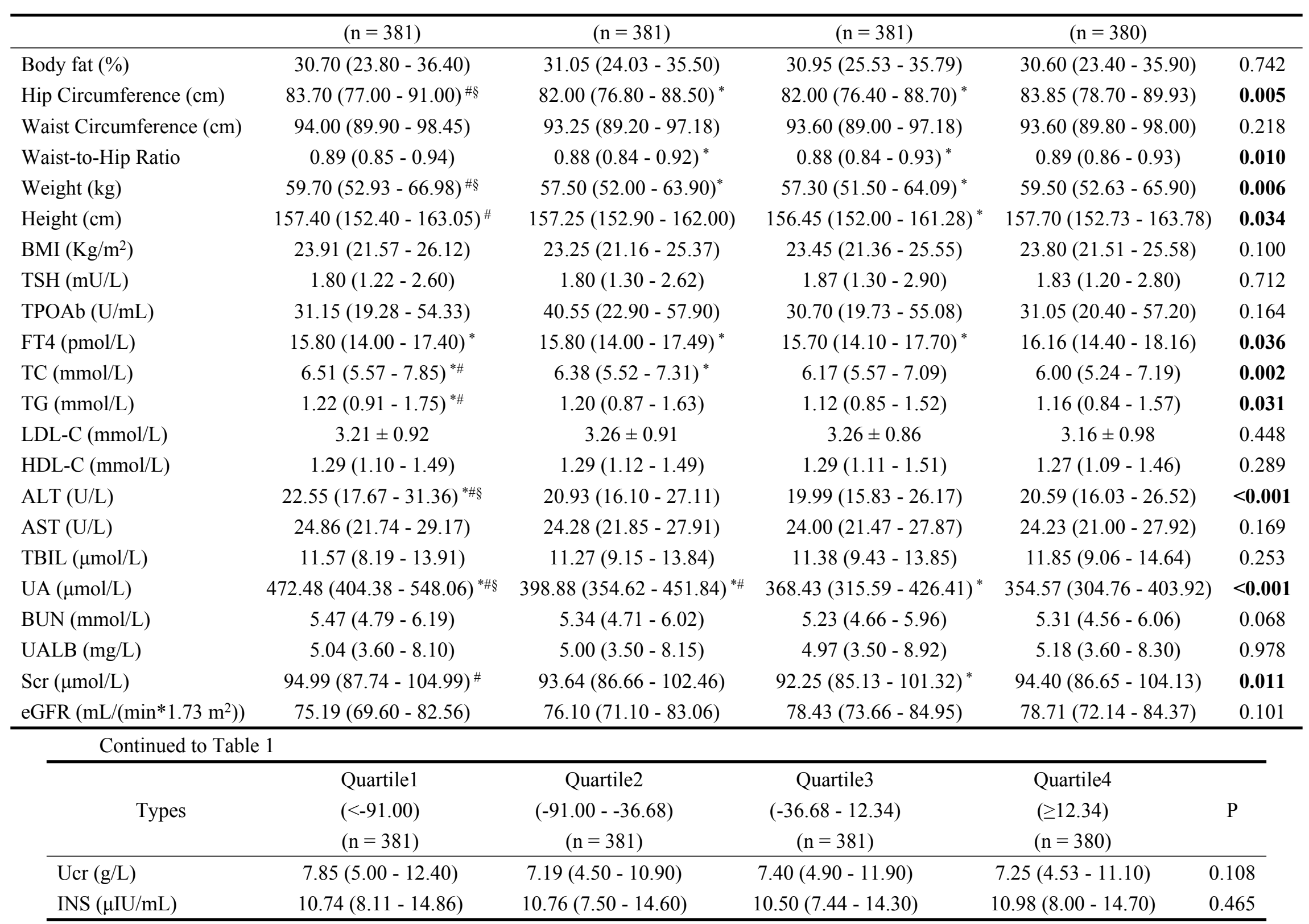


Data were means \pm SD or medians (interquartile ranges) for skewed variables or numbers (proportions) for categorical variables. $\mathrm{n}$, number of cases.

${ }^{*} \mathrm{P}<0.05$, statistically significance compared with Quartile 4 group.

\#P $<0.05$, statistically significance compared with Quartile 3 group.

$\S \mathrm{P}<0.05$, statistically significance compared with Quartile 2 group.

Fasting plasma glucose (FPG, mmol/L); 2 hour plasma glucose $(2 \mathrm{hPG}, \mathrm{mmol} / \mathrm{L})$; glycated hemoglobin A1c (HbA1c, \%); homeostasis model assessment of insulin resistance (HOMA-IR); systolic blood pressure (SBP, mmHg); diastolic blood pressure (DBP, $\mathrm{mmHg}$ ); body mass index (BMI, $\left.\mathrm{Kg} / \mathrm{m}^{2}\right)$; thyroid stimulating hormone (TSH, $\left.\mathrm{mU} / \mathrm{L}\right)$; thyroid peroxidase antibody (TPOAb, U/mL); free thyroxine (FT4, pmol/L); total cholesterol (TC, mmol/L); triglycerides (TG, mmol/L); low-density lipoprotein cholesterol (LDL-C, $\mathrm{mmol} / \mathrm{L}$ ); high-density lipoprotein cholesterol (HDL-C, mmol/L); alanine aminotransferase (ALT, U/L); aspartate aminotransferase (AST, $\mathrm{U} / \mathrm{L}$ ); total bilirubin (TBIL, $\mu \mathrm{mol} / \mathrm{L}$ ); uric acid (UA, $\mu \mathrm{mol} / \mathrm{L})$; blood urea nitrogen $(\mathrm{BUN}, \mathrm{mmol} / \mathrm{L}$ ); urine microalbumin (UALB, $\mathrm{mg} / \mathrm{L})$; serum creatinine $(\mathrm{Scr}, \mu \mathrm{mol} / \mathrm{L})$; estimated glomerular filtration rate $\left(\mathrm{eGFR}, \mathrm{mL} /\left(\min * 1.73 \mathrm{~m}^{2}\right)\right.$ ); urine creatinine $(\mathrm{Ucr}, \mathrm{g} / \mathrm{L})$; fasting serum insulin (INS, $\mu \mathrm{IU} / \mathrm{mL}$ ). 
Table 2 (on next page)

Prevalence of renal function decline according to changes of serum uric acid 
Table. 2 Hazard ratios of renal function decline according to changes of serum uric acid

\begin{tabular}{ccccccc}
\hline \multirow{2}{*}{ Types } & $\begin{array}{c}\text { Quartile1 } \\
(<-91.00) \\
(\mathrm{n}=374)\end{array}$ & $\begin{array}{c}\text { Quartile2 } \\
(-91.00--36.68) \\
(\mathrm{n}=371)\end{array}$ & $\begin{array}{c}\text { Quartile3 } \\
(-36.68-12.34) \\
(\mathrm{n}=373)\end{array}$ & $\begin{array}{c}\text { Quartile4 } \\
(\geq 12.34) \\
(\mathrm{n}=371)\end{array}$ & $\mathrm{P}$ \\
\hline \multirow{3}{*}{ Renal } & Model 1 & $0.52(0.39-0.69)$ & $0.62(0.48-0.80)$ & $0.72(0.56-0.92)$ & 1.00 & $<\mathbf{0 . 0 0 1}$ \\
function & Model 2 & $0.53(0.40-0.69)$ & $0.62(0.48-0.81)$ & $0.73(0.56-0.93)$ & 1.00 & $<\mathbf{0 . 0 0 1}$ \\
reduction & Model 3 & $0.56(0.42-0.73)$ & $0.65(0.50-0.84)$ & $0.74(0.58-0.95)$ & 1.00 & $<\mathbf{0 . 0 0 1}$ \\
& Model 5 & $0.55(0.42-0.73)$ & $0.66(0.50-0.85)$ & $0.75(0.58-0.96)$ & 1.00 & $\mathbf{0 . 0 0 2}$ \\
& $0.64(0.49-0.85)$ & $0.65(0.50-0.84)$ & $0.75(0.58-0.96)$ & 1.00 & $<\mathbf{0 . 0 0 1}$ \\
\hline
\end{tabular}

2 Data are hazard ratio (95\% confidence interval). Participants without renal function reduction are defined as 0

3 and with renal function reduction as 1 . $n$, cases.

4 Model 1 is unadjusted.

5 Model 2 is adjusted for sex and age.

6 Model 3 is adjusted for sex, age and dietary exercise intervention.

7 Model 4 is adjusted for sex, age, dietary exercise intervention, Waist-to-Hip Ratio and BMI.

8 Model 5 is adjusted for sex, age, dietary exercise intervention, Waist-to-Hip Ratio, BMI, pulse pressure 9 variation, $2 \mathrm{hPG}$ variation, AST variation, ALT variation, TC variation.

10 Body mass index (BMI, $\mathrm{Kg} / \mathrm{m} 2)$; 2 hour plasma glucose (2hPG, mmol/L); alanine aminotransferase (ALT, $11 \mathrm{U} / \mathrm{L}$ ); aspartate aminotransferase (AST, U/L); total cholesterol (TC, mmol/L). 


\section{Table 3(on next page)}

Prevalence of renal function improvement according to changes of serum uric acid in population with renal dysfunction 
Table. 3 Hazard ratios of renal function improvement according to changes of serum uric acid

\begin{tabular}{ccccccc}
\hline \multirow{2}{*}{ Types } & & $\begin{array}{c}\text { Quartile1 } \\
(<-91.00)\end{array}$ & $\begin{array}{c}\text { Quartile2 } \\
(-91.00--36.68)\end{array}$ & $\begin{array}{c}\text { Quartile3 } \\
(-36.68-12.34) \\
(\mathrm{n}=328)\end{array}$ & $\begin{array}{c}\text { Quartile4 } \\
(\geq 12.34) \\
(\mathrm{n}=340)\end{array}$ & $\begin{array}{c}\mathrm{P}=334) \\
(\mathrm{n}=317)\end{array}$ \\
\hline \multirow{3}{*}{ Renal } & Model 1 & $3.08(2.13-4.47)$ & $1.76(1.17-2.63)$ & $1.34(0.88-2.06)$ & 1.00 & $<\mathbf{0 . 0 0 1}$ \\
Function & Model 2 & $3.00(2.07-4.35)$ & $1.72(1.15-2.57)$ & $1.29(0.84-1.97)$ & 1.00 & $<\mathbf{0 . 0 0 1}$ \\
improvement 3 & $2.81(1.94-4.07)$ & $1.63(1.08-2.43)$ & $1.25(0.82-1.91)$ & 1.00 & $<\mathbf{0 . 0 0 1}$ \\
& Model 4 & $2.82(1.95-4.09)$ & $1.61(1.07-2.42)$ & $1.22(0.80-1.87)$ & 1.00 & $<\mathbf{0 . 0 0 1}$ \\
& Model 5 & $3.93(2.63-5.88)$ & $1.91(1.26-2.88)$ & $1.30(0.84-1.99)$ & 1.00 & $<\mathbf{0 . 0 0 1}$ \\
& Model 6 & $2.27(1.45-3.57)$ & $1.78(1.17-2.69)$ & $1.24(0.80-1.92)$ & 1.00 & $<\mathbf{0 . 0 0 1}$ \\
\hline
\end{tabular}

2 Data are hazard ratio (95\% confidence interval). Participants without renal function improvement are defined

3 as 0 and with renal function improvement as 1 . n, cases.

4 Model 1 is unadjusted.

5 Model 2 is adjusted for sex and age.

6 Model 3 is adjusted for sex, age and dietary exercise intervention.

7 Model 4 is adjusted for sex, age, dietary exercise intervention, BMI.

8 Model 5 is adjusted for sex, age, dietary exercise intervention, BMI, UA, HOMA-IR, HDL-C.

9 Model 6 is adjusted for sex, age, dietary exercise intervention, BMI, UA, HOMA-IR, HDL-C, HbAlc 10 variation, ALT variation, AST varaition.

11 Body mass index (BMI, $\mathrm{Kg} / \mathrm{m}^{2}$ ); uric acid (UA, $\left.\mu \mathrm{mol} / \mathrm{L}\right)$; homeostasis model assessment of insulin resistance 12 (HOMA-IR); high-density lipoprotein cholesterol (HDL-C, mmol/L); glycated hemoglobin A1c (HbA1c, \%); 13 alanine aminotransferase (ALT, U/L); aspartate aminotransferase (AST, U/L). 
Figure 1

Flow chart of the research subjects 
Community-based cohort in Guangzhou recruited 2876 subjects（in 2012）

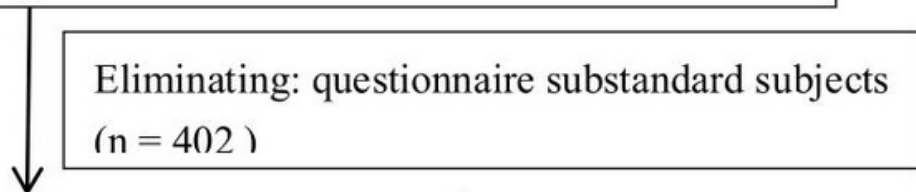

2474 subjects complete baseline survey in 2012

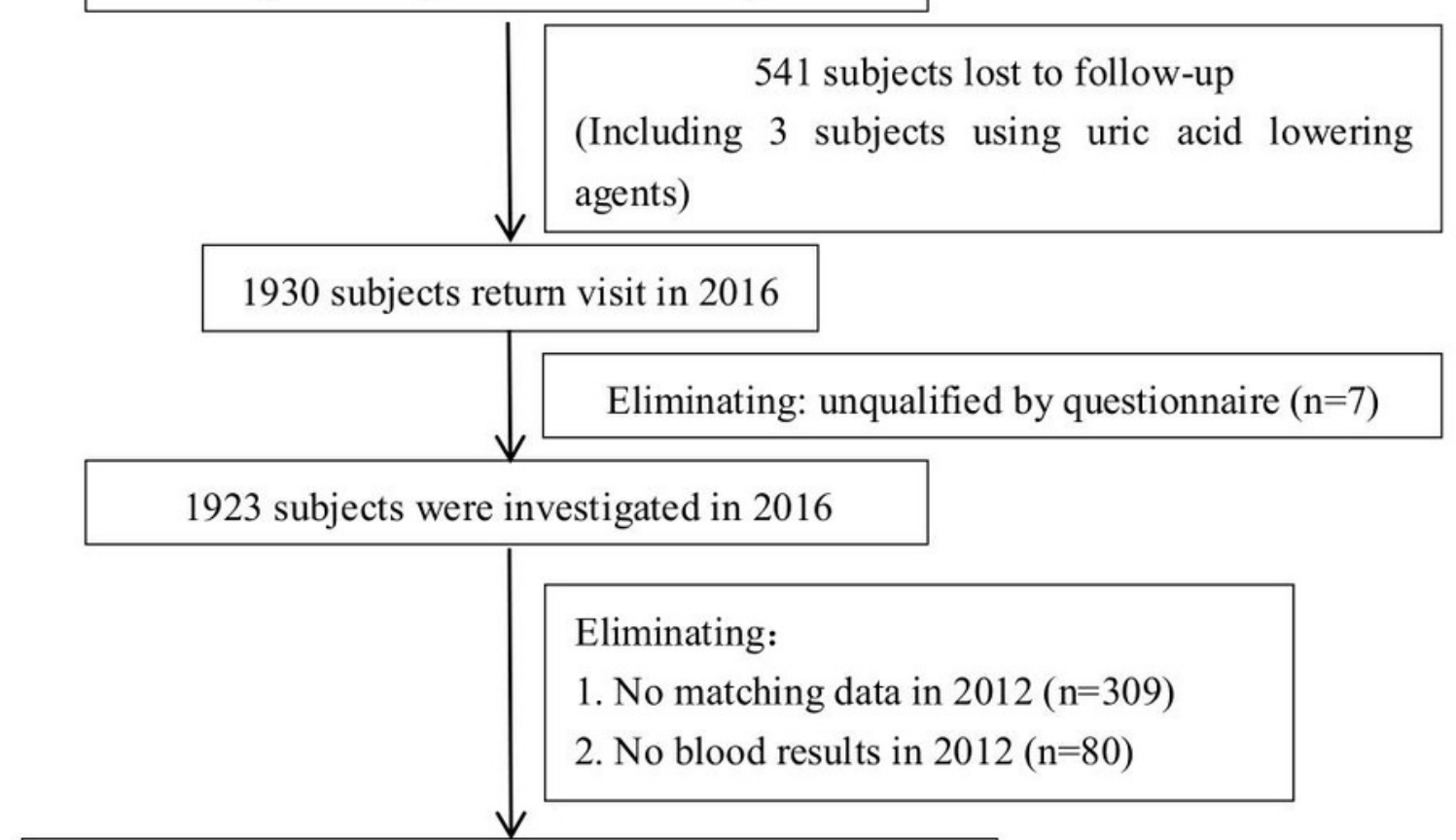

Totally 1534 subjects complete the follow-up until 2016

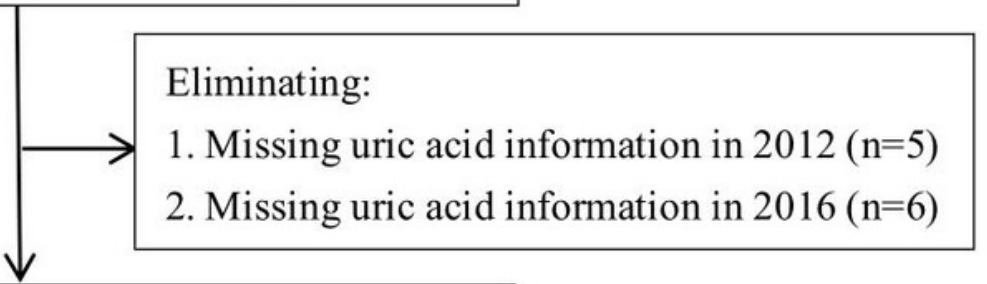

1523 subjects were included in the final analyses

1489 eligible individuals were included in the final analyses of association between uric acid lowering and renal function change 
Figure 2

Incidence of renal function decline or renal function improvement in different groups

(A) Renal function decline. (B) Renal function improvement.
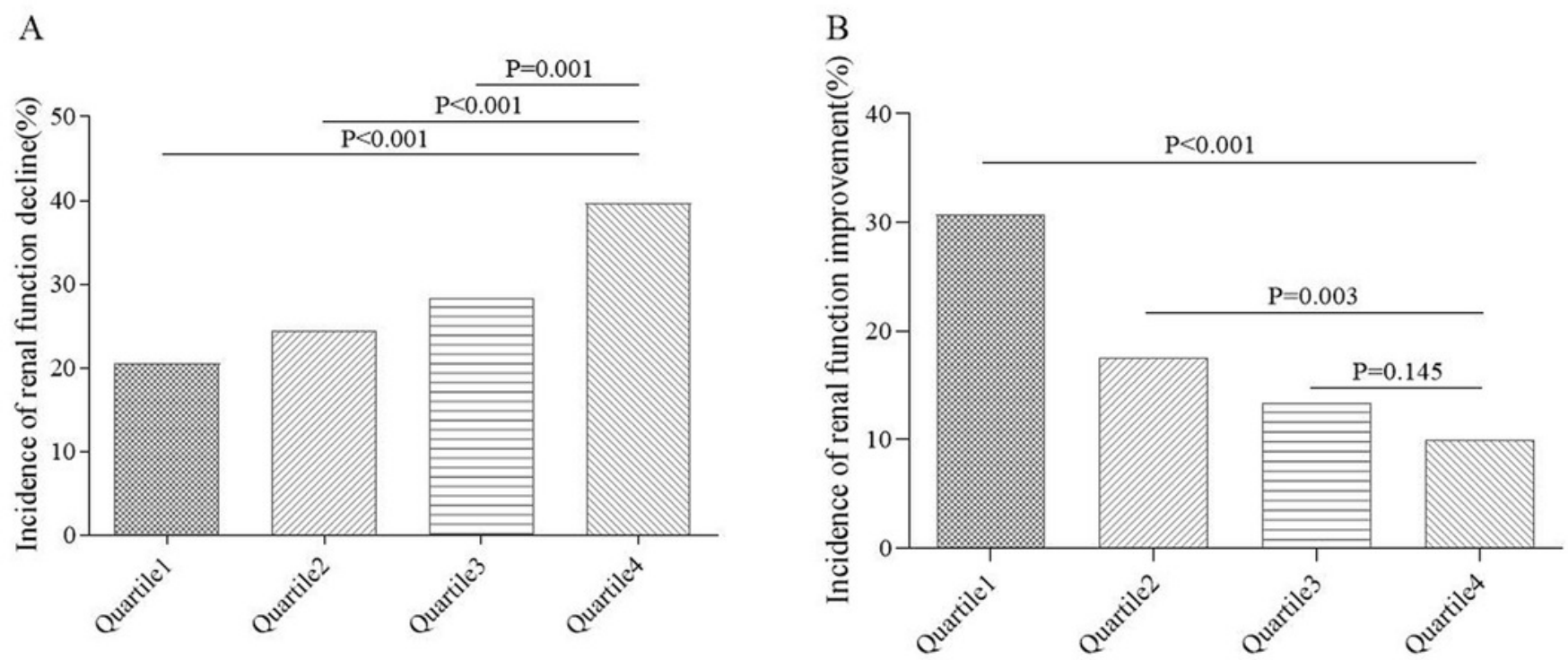
Figure 3

Prevalence of renal function decline with each quartile of changes in serum uric acid levels in the different subgroups

Sex
Male ( $n=396)$
Female $(n=1093)$
Age (Years)
$<60$ ( $n=999)$
$\geq 60$ ( $n=490)$
Diet and exercise intervention
Yes ( $n=682)$
No ( $n=807)$
Glucose tolerance status
NGT ( $n=394)$
Prediabetes( $n=852)$
Diabetes ( $n=231)$
High blood pressure
Yes ( $n=559)$
No $(n=921)$
Hyperuricemia
Yes ( $n=575)$
No $(n=914)$
BMI $(K g / m 2)$
$<24(n=827)$
$\geq 24$ ( $n=656)$

n, case/subjects

$121 / 396$

$300 / 1093$

$261 / 999$

$160 / 490$

$280 / 682$

$141 / 807$

$96 / 394$

268/394

$52 / 231$

$172 / 559$

248/921

$174 / 575$

$247 / 914$

$201 / 827$

$220 / 656$
RR $(95 \% \mathrm{Cl})$

1.21(1.09-1.35)

$1.21(0.94-1.30)$

$1.19(1.06-1.33)$

1.14(0.99-1.31)

1.13(1.01-1.26)

1.26(1.08-1.48)

$1.17(0.96-1.43)$

$1.13(1.01-1.26)$

$1.29(1.00-1.68)$

1.18(1.03-1.36)

1.18(1.05-1.33)

1.23(1.07-1.41)

$1.23(1.07-1.41)$

1.18(1.03-1.34)

1.14(1.01-1.29)
$P$ for interaction

0.061

0.379

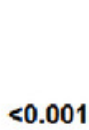

$<0.001$

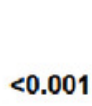

$<0.001$

0.011

0.002

0.375

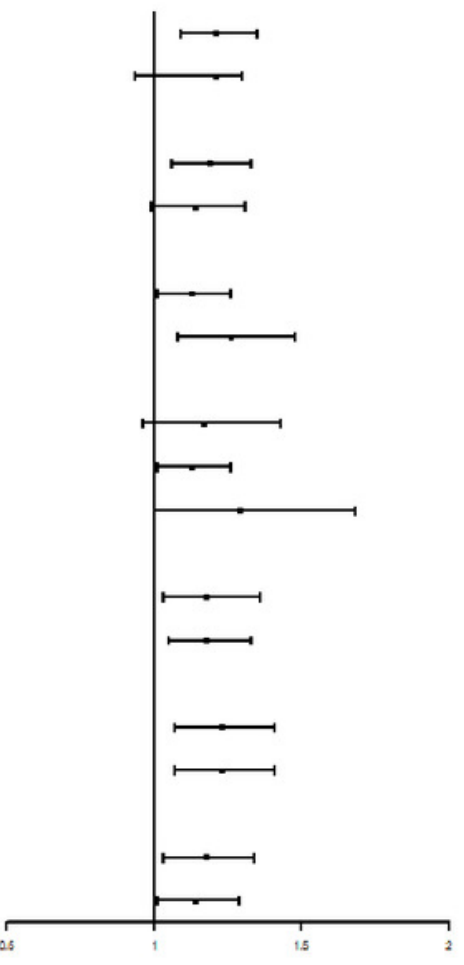


Figure 4

Prevalence of renal function improvement with each quartile of changes of serum uric acid levels in the different subgroups

Sex
Male ( $n=396)$
Female $(n=1093)$
Age (Years)
$<60$ ( $n=999)$
$\geq 60$ ( $n=490)$
Diet and exercise intervention
Yes ( $n=682)$
No ( $n=807)$
Glucose tolerance status
NGT ( $n=394)$
Prediabetes( $n=852)$
Diabetes ( $n=231)$
High blood pressure
Yes ( $n=559)$
No $(n=921)$
Hyperuricemia
Yes ( $n=575)$
No $(n=914)$
BMI (Kg/m2)
$<24$ ( $n=827)$
$\geq 24$ ( $n=656$ )

n, case/subjects

$121 / 396$

$300 / 1093$

$261 / 999$

$160 / 490$

$280 / 682$

141/807

$96 / 394$

268/852

$52 / 231$

$172 / 559$

248/921

$174 / 575$

247/914

201/827

$220 / 656$
RR $(95 \% \mathrm{Cl})$

$0.74(0.52-1.05)$

$0.76(0.65-0.89)$

$0.74(0.63-0.88)$

$0.79(0.61-1.03)$

0.89(0.67-1.18)

$0.73(0.61-1.86)$

$0.77(0.59-1.01)$

0.84(0.68-1.02)

$0.60(0.43-0.83)$

$0.77(0.60-1.00)$

$0.74(0.62-0.88)$

$0.76(0.59-0.99)$

$0.75(0.62-0.90)$

0.78(0.65-0.94)

0.73(0.59-0.92)
P for interaction

$<0.001$

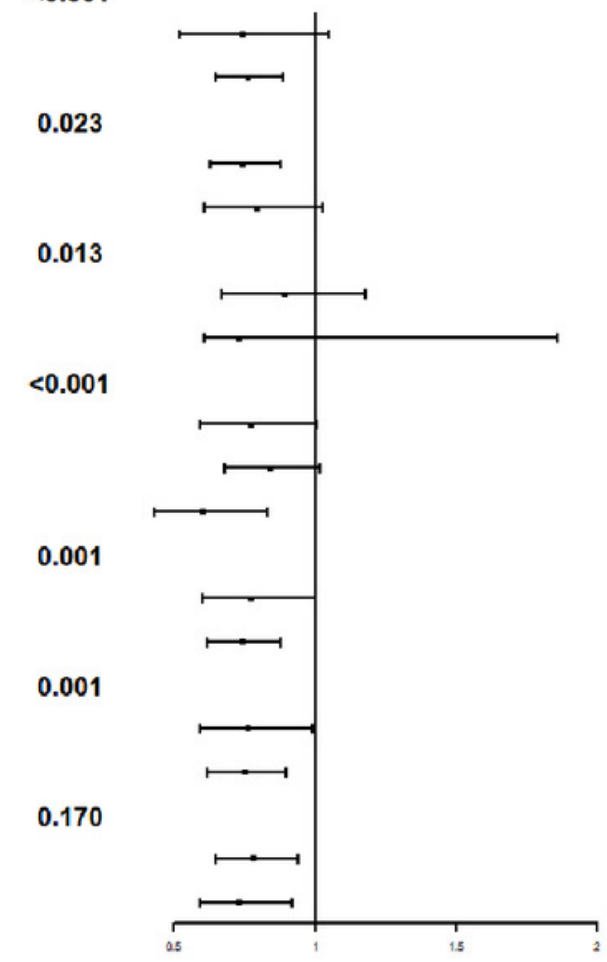

